\title{
A meta-analysis of epigenome-wide association studies in Alzheimer's disease highlights novel differentially methylated loci across cortex
}

\author{
Rebecca G. Smith ${ }^{1,19}$, Ehsan Pishva (10 1,2,19, Gemma Shireby' ${ }^{1}$, Adam R. Smith (1) 1, Janou A. Y. Roubroeks ${ }^{1,2}$, \\ Eilis Hannon (1) 1, Gregory Wheildon', Diego Mastroeni (10 3, Gilles Gasparoni (10 4, Matthias Riemenschneider ${ }^{5}$, \\ Armin Giese ${ }^{6}$, Andrew J. Sharp (1) 7, Leonard Schalkwyk (1) ${ }^{8}$, Vahram Haroutunian (D) 9,10,11, \\ Wolfgang Viechtbauer (1) ${ }^{2}$, Daniel L. A. van den Hove 2,12, Michael Weedon', Danielle Brokaw ${ }^{3}$, Paul T. Francis', \\ Alan J. Thomas ${ }^{13}$, Seth Love ${ }^{14}$, Kevin Morgan (1) ${ }^{15}$, Jörn Walter (1) ${ }^{4}$, Paul D. Coleman ${ }^{3}$, David A. Bennett ${ }^{16}$, \\ Philip L. De Jager (iD) ${ }^{17,18}$, Jonathan Mill (i) ${ }^{1} \&$ Katie Lunnon (1) ${ }^{1 凶}$
}

Epigenome-wide association studies of Alzheimer's disease have highlighted neuropathologyassociated DNA methylation differences, although existing studies have been limited in sample size and utilized different brain regions. Here, we combine data from six DNA methylomic studies of Alzheimer's disease ( $N=1453$ unique individuals) to identify differential methylation associated with Braak stage in different brain regions and across cortex. We identify $236 \mathrm{CpGs}$ in the prefrontal cortex, $95 \mathrm{CpGs}$ in the temporal gyrus and ten CpGs in the entorhinal cortex at Bonferroni significance, with none in the cerebellum. Our crosscortex meta-analysis ( $N=1408$ donors) identifies $220 \mathrm{CpGs}$ associated with neuropathology, annotated to 121 genes, of which 84 genes have not been previously reported at this significance threshold. We have replicated our findings using two further DNA methylomic datasets consisting of a further $>600$ unique donors. The meta-analysis summary statistics are available in our online data resource (www.epigenomicslab.com/ad-meta-analysis/).

\footnotetext{
${ }^{1}$ University of Exeter Medical School, College of Medicine and Health, University of Exeter, Exeter, UK. ${ }^{2}$ Department of Psychiatry and Neuropsychology, School for Mental Health and Neuroscience (MHeNS), Maastricht University, Maastricht, The Netherlands. ${ }^{3}$ Banner ASU Neurodegenerative Research Center, Biodesign Institute, Arizona State University, Tempe, AZ, USA. ${ }^{4}$ Department of Genetics, University of Saarland (UdS), Saarbruecken, Germany. ${ }^{5}$ Department of Psychiatry and Psychotherapy, Saarland University Hospital (UKS), Homburg, Germany. ${ }^{6}$ Center for Neuropathology and Prion Research, Ludwig-Maximilians-University (LMU), Munich, Germany. ${ }^{7}$ Department of Genetics and Genomic Sciences, Icahn School of Medicine at Mount Sinai, New York, NY, USA. ${ }^{8}$ School of Biological Sciences, University of Essex, Colchester, UK. ${ }^{9}$ Department of Psychiatry, The Icahn School of Medicine at Mount Sinai, New York, NY, USA. ${ }^{10}$ Department of Neuroscience, The Icahn School of Medicine at Mount Sinai, New York, NY, USA. ${ }^{11}$ JJ Peters VA Medical Center, Bronx, NY, USA. ${ }^{12}$ Laboratory of Translational Neuroscience, Department of Psychiatry, Psychosomatics and Psychotherapy, University of Wuerzburg, Würzburg, Germany. ${ }^{13}$ Institute of Neuroscience, Newcastle University, Newcastle Upon Tyne, UK. ${ }^{14}$ Dementia Research Group, Institute of Clinical Neurosciences, School of Clinical Sciences, University of Bristol, Bristol, UK. ${ }^{15}$ Human Genetics Group, University of Nottingham, Nottingham, UK. ${ }^{16}$ Rush Alzheimer's Disease Center, Rush University Medical Center, Chicago, IL, USA. ${ }^{17}$ Center for Translational and Computational Neuroimmunology, Department of Neurology and Taub Institute, Columbia University Medical Center, New York, NY, USA. ${ }^{18}$ The Broad Institute of MIT and Harvard, Cambridge, MA, USA. ${ }^{19}$ These authors contributed equally: Rebecca G. Smith, Ehsan Pishva. ${ }^{凶}$ email: k.lunnon@exeter.ac.uk
} 
$\Lambda$ discisis lzheimer's disease $(\mathrm{AD})$ is a chronic neurodegenerative disease that is accompanied by memory problems, confusion and changes in mood, behavior and personality. $\mathrm{AD}$ accounts for $\sim 60 \%$ of dementia cases, which affected 43.8 million people worldwide in $2016^{1}$. The disease is defined by two key pathological hallmarks in the brain: extracellular plaques comprised of amyloid-beta protein and intracellular neurofibrillary tangles of hyperphosphorylated tau protein ${ }^{2-4}$. These neuropathological changes are thought to occur perhaps decades before clinical symptoms manifest and the disease is diagnosed ${ }^{4}$. $\mathrm{AD}$ is a multi-factorial and complex disease, with the risk of developing disease still largely unknown despite numerous genetic and epidemiological studies over recent years.

Several studies have suggested that epigenetic mechanisms may play a role in disease etiology. In recent years a number of epigenome-wide association studies (EWAS) have been performed in $\mathrm{AD}$ brain samples, which have predominantly utilized the Illumina Infinium HumanMethylation450K BeadChip (450K array) in conjunction with bisulfite-treated DNA to assess levels of DNA methylation in cortical brain tissue from donors with varying degrees of $\mathrm{AD}$ pathology ${ }^{5-12}$. Independently these studies have identified a number of loci that show robust differential DNA methylation in disease, and many of these overlap between studies, for example, loci annotated to ANK1, RHBDF2, HOXA3, CDH23 and RPL13 have been consistently reported.

Here we have performed a meta-analysis of six independent existing EWAS of AD brain ${ }^{5-8,10,12}$, totalling 1453 independent donors, to identify robust and consistent differentially methylated loci associated with Braak stage, used as a measure of neurofibrillary tangle spread through the brain, in different brain regions and across the cortex. In our intra-tissue meta-analysis we identify $236 \mathrm{CpGs}$ in the prefrontal cortex ( $N=961$ samples), 95 in the temporal gyrus $(N=608$ samples) and ten in the entorhinal cortex ( $N=189$ samples) at Bonferroni significance, with none in the cerebellum ( $N=533$ samples). Our cross-cortex meta-analysis ( $N=1408$ individuals) identified 220 Bonferroni significant CpGs, which were replicated in two further independent DNA methylation datasets. Our meta-analysis approach provides additional power to detect DNA methylomic variation associated with AD pathology at novel loci, in addition to providing further replication of loci that have been previously identified in the smaller independent EWAS.

\section{Results}

Pathology-associated DNA methylation signatures in discrete cortical brain regions. We identified six EWAS of DNA methylation in $\mathrm{AD}$ that had been generated using the $450 \mathrm{~K}$ array and had a cohort size of $>50$ unique donors. All had data on Braak stage available, which we used as a standardized measure of tau pathology spread through the brain (Table 1). We were interested in identifying epigenomic profiles associated with Braak stage in specific brain regions, leveraging additional power by meta-analysing multiple studies to identify novel loci. To this end, we performed an EWAS in each available tissue and cohort separately, looking for an association between DNA methylation and Braak stage, whilst controlling for age and sex (all tissues) and neuron/glia proportion (cortical bulk tissues only), with

Table 1 Demographic information for cohorts included in the meta-analyses.

\begin{tabular}{|c|c|c|c|c|c|c|c|c|}
\hline Stage & Cohort & $\begin{array}{l}\text { Unique } \\
\text { individuals }\end{array}$ & $\begin{array}{l}\text { Ancestry (Eu/ } \\
\text { Af/Am/As) }\end{array}$ & Braak & Number & $\operatorname{Sex}(M / F)$ & $\begin{array}{l}\text { Age at death } \\
\text { in }( \pm S D)\end{array}$ & Tissues analysed \\
\hline \multirow[t]{18}{*}{ Discovery } & \multirow[t]{3}{*}{ London 1} & \multirow[t]{3}{*}{113} & \multirow[t]{3}{*}{$112 / 0 / 1 / 0$} & $0-11$ & 29 & $13 / 16$ & $77.6(12.8)$ & \multirow{6}{*}{$\begin{array}{l}\text { Prefrontal cortex, entorhinal cortex, } \\
\text { superior temporal gyrus, } \\
\text { cerebellum (Bulk) } \\
\text { Entorhinal cortex, cerebellum (Bulk) }\end{array}$} \\
\hline & & & & III-IV & 18 & $7 / 11$ & $88.5(5.2)$ & \\
\hline & & & & $\mathrm{V}-\mathrm{VI}$ & 66 & $26 / 40$ & $85.4(8.1)$ & \\
\hline & \multirow[t]{3}{*}{ London 2} & \multirow[t]{3}{*}{95} & \multirow[t]{3}{*}{$92 / 1 / 2 / 0$} & $0-11$ & 23 & $12 / 11$ & $76.1(10.0)$ & \\
\hline & & & & III-IV & 16 & $3 / 13$ & $87.6(6.4)$ & \\
\hline & & & & $\mathrm{V}-\mathrm{VI}$ & 56 & $26 / 30$ & $81.5(8.6)$ & \\
\hline & \multirow[t]{3}{*}{ Mount Sinai } & \multirow[t]{3}{*}{146} & \multirow[t]{3}{*}{$113 / 20 / 11 / 2$} & $0-11$ & 60 & $32 / 28$ & $82(7.6)$ & \multirow{3}{*}{$\begin{array}{l}\text { Prefrontal cortex, superior temporal } \\
\text { gyrus (Bulk) }\end{array}$} \\
\hline & & & & III-IV & 42 & $12 / 30$ & $88.8(6.6)$ & \\
\hline & & & & V-VI & 44 & $12 / 32$ & $88.0(7.5)$ & \\
\hline & \multirow[t]{3}{*}{ Arizona 1} & \multirow[t]{3}{*}{302} & \multirow[t]{3}{*}{$302 / 0 / 0 / 0$} & $0-11$ & 61 & $40 / 21$ & $80.3(8.2)$ & \multirow{3}{*}{$\begin{array}{l}\text { Middle temporal gyrus, } \\
\text { cerebellum (Bulk) }\end{array}$} \\
\hline & & & & III-IV & 97 & $50 / 47$ & $86.9(6.9)$ & \\
\hline & & & & $\mathrm{V}-\mathrm{VI}$ & 144 & $63 / 81$ & $82.3(8.5)$ & \\
\hline & \multirow[t]{3}{*}{ Arizona 2} & \multirow[t]{3}{*}{88} & \multirow[t]{3}{*}{$88 / 0 / 0 / 0$} & $0-11$ & 16 & $10 / 6$ & $82.5(5.0)$ & \multirow{3}{*}{$\begin{array}{l}\text { Middle temporal gyrus, } \\
\text { cerebellum (Bulk) }\end{array}$} \\
\hline & & & & III-IV & 45 & $21 / 24$ & $86.7(5.1)$ & \\
\hline & & & & $\mathrm{V}-\mathrm{VI}$ & 27 & $12 / 15$ & $84.6(7.1)$ & \\
\hline & \multirow[t]{3}{*}{ ROS/MAP } & \multirow[t]{3}{*}{711} & \multirow[t]{3}{*}{$711 / 0 / 0 / 0$} & $0-11$ & 143 & $70 / 73$ & $83.2(6.0)$ & \multirow[t]{3}{*}{ Prefrontal cortex (Bulk) } \\
\hline & & & & III-IV & 410 & $144 / 266$ & $86.9(4.1)$ & \\
\hline & & & & $\mathrm{V}-\mathrm{VI}$ & 158 & $45 / 113$ & $87.8(3.5)$ & \\
\hline \multirow[t]{9}{*}{ Replication } & \multirow[t]{6}{*}{ Munich } & \multirow[t]{3}{*}{45} & \multirow[t]{3}{*}{-} & $0-11$ & 9 & $5 / 4$ & $76.7(10.9)$ & \multirow[t]{3}{*}{ Prefrontal cortex (Bulk) } \\
\hline & & & & III-IV & 7 & $1 / 6$ & $82.1(5.2)$ & \\
\hline & & & & $\mathrm{V}-\mathrm{VI}$ & 29 & $12 / 17$ & $79.2(8.5)$ & \\
\hline & & \multirow[t]{3}{*}{26} & \multirow[t]{3}{*}{-} & $0-11$ & 11 & $7 / 4$ & $75.9(8.5)$ & Occipital cortex (Sorted cells) \\
\hline & & & & III-IV & 5 & $1 / 4$ & $85.0(6.5)$ & \\
\hline & & & & $\mathrm{V}-\mathrm{VI}$ & 10 & $4 / 6$ & $77.9(6.6)$ & \\
\hline & BDR & 590 & - & $0-11$ & 196 & $100 / 96$ & $83.6(10.6)$ & Prefrontal cortex (Bulk) \\
\hline & & & & III-IV & 136 & $80 / 56$ & $85.1(7.45)$ & \\
\hline & & & & $\mathrm{V}-\mathrm{VI}$ & 258 & $128 / 130$ & $82.5(8.5)$ & \\
\hline
\end{tabular}

Sample numbers, split of males (M)/females (F) and mean age at death in years ( \pm standard deviation [SD]) are shown for individuals with low pathology (Braak 0-II), mid-stage pathology (Braak III-IV) and severe pathology (Braak V-VI) in each cohort. Shown are the bulk tissues available from each cohort, which included the cerebellum, entorhinal cortex, middle temporal gyrus, prefrontal cortex and superior temporal gyrus. In the discovery meta-analyses, we used data from six EWAS using the 450K array, which all had >50 unique donors. For replication we used two cohorts. The Munich cohort had 450K data from bulk prefrontal cortex tissue, as well as data available from sorted neuronal and non-neuronal cell populations from the occipital cortex. The BDR cohort had EPIC array data available from bulk prefrontal cortex samples. For the meta-analyses, superior temporal gyrus and middle temporal gyrus samples were both classed as temporal gyrus samples. Shown are final numbers for all cohorts after data quality control. Ancestry is reported for the discovery cohorts and is the number of unique individuals that had the following inferred ethnicities from the 1000 genomes reference panel: European (Eu), African (Af), American (Am), East Asian (As). 
surrogate variables added as appropriate to reduce inflation. For discovery, we then used the estimated effect size (ES) and standard errors (SEs) from these six studies $(N=1453$ unique donors) for a fixed-effect inverse variance weighted meta-analysis separately for each tissue (prefrontal cortex: three cohorts, $N=$ 961; temporal gyrus: four cohorts, $N=608$, entorhinal cortex: two cohorts, $N=189$ cerebellum: four cohorts, $N=533$ ) (Supplementary Fig. 1).

The prefrontal cortex represented our largest dataset $(N=$ 961 samples) and we identified 236 Bonferroni significant differentially methylated positions (DMPs) $\left(P<1.238 \times 10^{-7}\right.$ to account for 403,763 probes), of which 193 were annotated to 137 genes, with 43 unannotated loci based on Illumina UCSC annotation (Fig. 1a, Supplementary Fig. 2, Supplementary Data 1). Previous EWAS of the prefrontal cortex have consistently reported the HOXA gene cluster as a region that is hypermethylated in $\mathrm{AD}^{6,7}$, with a cell-type specific EWAS demonstrating this is neuronal-derived ${ }^{11}$. Indeed, the most significant DMP in the prefrontal cortex in our meta-analysis resided in HOXA3 (cg22962123: ES [defined as the methylation difference between Braak 0 and Braak VI] $=0.042, P=5.97 \times 10^{-15}$ ), with a further 16 of the Bonferroni significant DMPs also annotated to this gene. This locus appeared to be particularly hypermethylated with higher Braak stage in the prefrontal cortex, and to a slightly lesser extent in the temporal gyrus (Supplementary Fig. 3). There was no significant difference in methylation at this locus in the entorhinal cortex $(P=0.864)$, which is interesting given that the entorhinal cortex may succumb to pathology early in the disease process (Braak stage III). Of the 236 prefrontal cortex DMPs, $92 \%$ (217 probes) were nominally significant $(P<0.05)$ in the temporal gyrus, of which $12 \%$ (28 probes) were Bonferroni significant, whilst $9 \%$ (22 probes) were nominally significant in the entorhinal cortex, with $1 \%$ ( 3 probes) reaching Bonferroni significance (Fig. 1b). The effect sizes for the 236 Bonferroni significant prefrontal cortex DMPs were correlated with the effect sizes for the same probes in both the temporal gyrus (Pearson's correlation coefficient $\left.(r)=0.94, P=6.17 \times 10^{-112}\right)$ and entorhinal cortex $\left(r=0.58, P=1.80 \times 10^{-22}\right)$ and were enriched for probes with the same direction of effect (sign test: temporal gyrus $P=5.07 \times 10^{-67}$, entorhinal cortex $P=6.88 \times 10^{-26}$ ) (Supplementary Fig. 4). For the 236 Bonferroni significant prefrontal cortex DMPs these had the largest effect sizes in the prefrontal cortex, with a smaller effect size in the temporal gyrus and entorhinal cortex (Fig. 1c). Of these 236 DMPs, 29 of these had being previously reported at Bonferroni significance in previous publications on the individual cohorts ${ }^{5-7,12}$, including one probe annotated to $A N K 1$, one probe annotated to HOXA3, one probe annotated to PPT2/PRRT1 and two probes annotated to RHBDF2, amongst others. However, our approach has identified 207 novel Bonferroni significant DMPs (although several had been reported in previous studies at a more relaxed significance threshold, or in regional analyses). This included several additional probes residing in genes already identified (from another probe) in earlier studies, for example, a further 16 probes in HOXA3 and two probes in PPT2/PRRT1. Interestingly, we also identified a number of novel genes, including some which featured multiple Bonferroni significant DMPs including for example, seven probes in AGAP2 and five probes in SLC44A2, amongst others. One other noteworthy novel Bonferroni significant DMP in the prefrontal cortex was cg08898775 $\left(\mathrm{ES}=0.019, P=4.03 \times 10^{-9}\right)$, annotated to $A D A M 10$, which encodes for $\alpha$-secretase which cleaves APP in the non-amyloidogenic pathway. A differentially methylated region (DMR) analysis, which allowed us to identify areas of the genome consisting of $\geq 2$ DMPs, revealed 262 significant DMRs in the prefrontal cortex (Supplementary Data 2), the most significant containing 20 probes and located in HOXA3 (chr7:27,153,212-27,155,234: Sidak-corrected $P=8.21 \times 10^{-50}$, Supplementary Fig. 5), as well as several other DMRs in the HOXA gene cluster.

A meta-analysis of temporal gyrus EWAS datasets $(N=$ 608 samples) identified 95 Bonferroni significant probes, of which 75 were annotated to 53 genes, with 20 unannotated probes using Illumina UCSC annotation (Fig. 1a, Supplementary Fig. 6, Supplementary Data 3). The most significant probe was cg11823178 (ES $=0.029, P=3.97 \times 10^{-16}$, Supplementary Fig. 7$)$, which is annotated to the ANK1 gene, with the fifth (cg05066959: $\left.\mathrm{ES}=0.042, P=4.58 \times 10^{-13}\right)$ and $82^{\text {nd }}(\operatorname{cg} 16140558: \mathrm{ES}=0.013, P$ $=8.44 \times 10^{-8}$ ) most significant probes in the temporal gyrus also being annotated to nearby CpGs in that gene. This locus has been widely reported to be hypermethylated in $\mathrm{AD}$ from prior EWAS $5,6,8,12$, as well as in other neurodegenerative diseases such as Huntington's disease and Parkinson's disease ${ }^{13}$. Another noteworthy gene is RHBDF2, where five Bonferroni significant DMPs in the temporal gyrus were annotated to (cg05810363: $\mathrm{ES}=$ $0.029, P=2.25 \times 10^{-11}$; $\operatorname{cg} 13076843: \mathrm{ES}=0.031, P=2.97 \times 10^{-11}$; cg09123026: $\mathrm{ES}=0.012, P=3.46 \times 10^{-9} ; \operatorname{cg} 12163800: \mathrm{ES}=0.025$, $\left.P=5.85 \times 10^{-9} ; \quad \operatorname{cg} 12309456: \quad \mathrm{ES}=0.016, P=1.33 \times 10^{-8}\right) ;$ and which has been highlighted in previous EWAS in $\mathrm{AD}$ in the individual cohorts ${ }^{5,6,12}$. Of the 95 Bonferroni significant DMPs in the temporal gyrus, $88 \%$ (84 probes) were nominally significant in the prefrontal cortex, of which $29 \%$ (28 probes) were Bonferroni significant, whilst $54 \%$ (51 probes) were nominally significant in the entorhinal cortex, of which 6\% (6 probes) were Bonferroni significant (Fig. 1b). Given the high degree of overlapping significant loci between the temporal gyrus and other cortical regions, it was not surprising that the ES of the 95 Bonferroni significant temporal gyrus probes were highly correlated with the ES of the same loci in both the prefrontal cortex $(r=0.91, P=5.09 \times$ $\left.10^{-38}\right)$ and entorhinal cortex $\left(r=0.77, P=4.02 \times 10^{-20}\right)$ and were enriched for the same direction of effect (sign test: prefrontal cortex $P=5.05 \times 10^{-29}$, entorhinal cortex $=2.30 \times 10^{-25}$ ) (Supplementary Fig. 8). The majority of the 95 Bonferroni significant DMPs in the temporal gyrus were hypermethylated, and the mean ES was greater in the temporal gyrus than the prefrontal cortex or entorhinal cortex (Fig. 1c). Thirty-two of the 95 Bonferroni significant DMPs in the temporal gyrus have been previously reported to be significantly differentially methylated in published EWAS, including for example, three probes in ANK1 and the five probes in RHBDF2. Our meta-analysis approach in the temporal gyrus has identified 63 novel DMPs (at Bonferroni significance), including some novel genes with multiple DMPs, for example, four probes in RGMA and two probes in CCND1, amongst others. Finally, our regional analysis highlighted 104 DMRs (Supplementary Data 4); the top DMR resided in the ANK1 gene (chr8:41,519,308-41,519,399) and contained two probes (Sidakcorrected $P=1.72 \times 10^{-21}$ ) (Supplementary Fig. 9). The five DMPs in $R H B D F 2$ that we already highlighted also represented a significant DMR (Sidak-corrected $P=8.47 \times 10^{-21}$ ), with three other genomic regions containing large, significant DMRs consisting of $\geq 10$ probes, such as MCF2L (chr13:113698408-113699016 [10 probes], Sidak-corrected $\left.P=1.16 \times 10^{-19}\right), \quad$ PRRT1/PPT2 (chr6:32120773-32121261 [17 probes], Sidak-corrected $P=4.90$ $\times 10^{-15}$ ) and HOXA5 (chr7:27184264-27184521 [10 probes], Sidakcorrected $\left.P=1.60 \times 10^{-7}\right)$.

The final cortical region we had available was the entorhinal cortex $(N=189)$, where we identified ten Bonferroni significant probes in our meta-analysis, all of which were hypermethylated with higher Braak stage (Fig. 1a, Supplementary Fig. 10, Supplementary Data 5). These ten probes were annotated to eight genes (Illumina UCSC annotation), with two Bonferroni significant probes residing in each of the ANK1 and SLC15A4 genes. As with the temporal gyrus, the most significant DMP was 
a
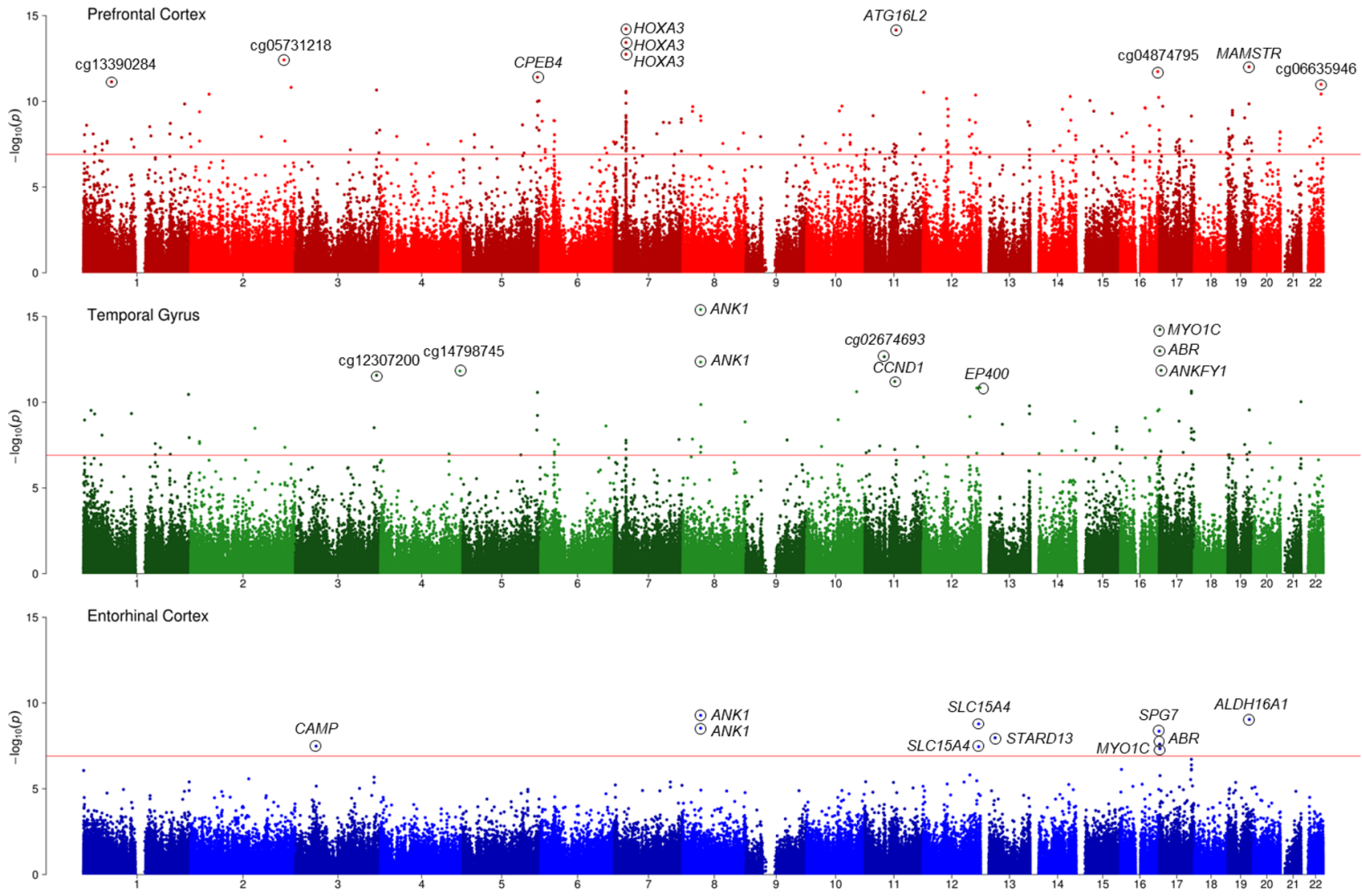

b

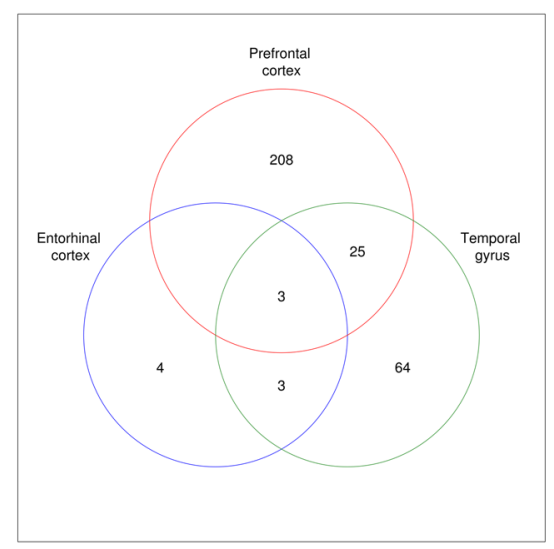

C

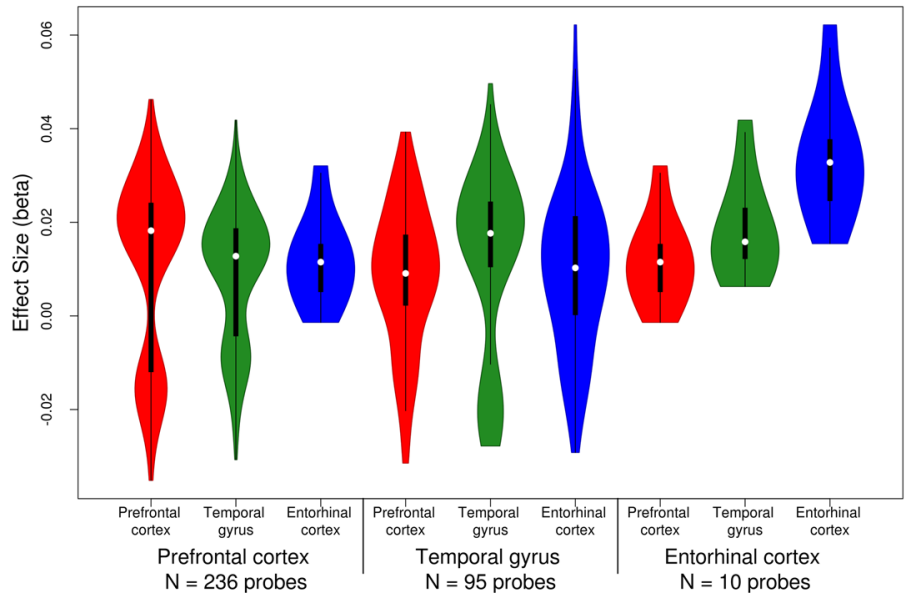

Fig. 1 Intra-tissue meta-analyses of AD methylomic studies highlights Bonferroni significant differentially methylated positions (DMPs) in all cortical tissues. a A Manhattan plot for the prefrontal cortex (red, $N=961$ ), temporal gyrus (green, $N=608$ ) and entorhinal cortex (blue, $N=189$ ) meta-analyses, with the ten most significant DMPs circled on the plot and Illumina UCSC gene name shown if annotated, or CpG ID if unannotated. The $X$-axis shows chromosomes 1-22 and the $Y$-axis shows $-\log 10(p)$, with the horizontal red line denoting Bonferroni significance $\left(P<1.238 \times 10^{-7}\right)$. b A Venn diagram highlighting overlapping DMPs at Bonferroni significance across the cortical tissues. $\mathbf{c}$ In each cortical brain region the Bonferroni significant DMPs identified in that region usually had a greater effect size (ES) there, than in any of the other cortical regions. The $X$-axis represents the methylation (beta) ES between individuals that are Braak stage $O$ and VI. Data are separated on the $Y$-axis by tissue analysis (large text) with the corresponding data at these probes in other tissues (small text). The white dot in the centre represents the median, the dark box represents the interquartile range (IQR), whilst the whisker lines represent the "minimum" (quartile 1-1.5 $\times I Q R$ ) and the 'maximum' (quartile $3+1.5 \times I Q R$ ). The coloured violin represents all samples including outliers, meaning that the 'minimum' and 'maximum' may not extend to the end of the violin. 
$\operatorname{cg} 11823178\left(\mathrm{ES}=0.045, P=5.22 \times 10^{-10}\right.$, Supplementary Fig. 7$)$, located within the $A N K 1$ gene, with the fourth most significant DMP being located within $100 \mathrm{bp}$ of that CpG (cg05066959: $\left.\mathrm{ES}=0.062, P=2.93 \times 10^{-9}\right)$. In total, eight of the ten DMPs in the entorhinal cortex had been reported previously at Bonferroni significance, including the two probes in ANK1. Two of the Bonferroni significant DMPs we identified in the entorhinal cortex were novel CpGs (cg11563844: STARD13, cg04523589: $C A M P)$, having not been reported as Bonferroni significant in previous EWAS. Of the ten entorhinal cortex probes, $90 \%$ (9 probes) were nominally significant in the temporal gyrus, of which $60 \%$ (6 probes) were Bonferroni significant, whilst $70 \%$ (7 probes) were nominally significant in the prefrontal cortex, of which $30 \%$ (3 probes) were Bonferroni significant (Fig. 1b). Of the four DMPs that were Bonferroni significant in only the entorhinal cortex, three of these were nominally significant in at least one other tissue, with just one probe unique to the entorhinal cortex, annotated to STARD13 (cg11563844, ES = $\left.0.027, P=1.07 \times 10^{-8}\right)$. The effect sizes of the ten Bonferroni significant DMPs in the entorhinal cortex were significantly correlated with the effect size of the same probes in the prefrontal cortex $(r=0.74, P=0.01)$ and temporal gyrus $(r=0.85, P=$ $\left.1.52 \times 10^{-3}\right)$ and were enriched for the same direction of effect (sign test: prefrontal cortex $P=0.021$, temporal gyrus $P=1.95 \times 10^{-3}$ ) (Supplementary Fig. 11). The ten DMPs were hypermethylated in all three cortical regions, with the greatest Braak-associated ES in the entorhinal cortex (Fig. 1c). A regional analysis identified seven DMRs (Supplementary Data 6); the top three DMRs (RHBDF2: chr17:74,475,240-74,475,402 [five probes], $P=7.68 \times 10^{-14}$, Supplementary Fig. 12 ; ANK1: chr8:41 519308-41519399 [two probes], $P=4.89 \times 10^{-13}$; SLC15A4: chr12:129281444-129281546 [three probes], $P=5.24 \times 10^{-12}$ ) were significant in at least one of the other cortical regions we meta-analysed.

To date, a few independent EWAS in $\mathrm{AD}$ have been undertaken in the cerebellum and none of these have reported any Bonferroni significant DMPs. In our meta-analysis we identified no Bonferroni significant DMPs, nor any DMRs in the cerebellum (Supplementary Fig. 13), despite this analysis including 533 independent samples. There was no correlation of the ES for the Bonferroni significant DMPs we had identified in the meta-analyses of the three cortical regions with the ES of the same probes in the cerebellum (prefrontal cortex: $r=0.11, P=$ 0.08 ; temporal gyrus: $r=0.14, P=0.17$; entorhinal cortex: $r=$ $0.48, P=0.16$; Supplementary Fig. 14).

220 CpGs are differentially methylated across the cortex in AD. We were interested in combining data from across the different cortical tissues to identify common differentially methylated loci across the cortex and also to provide more power by utilizing data from 1408 unique individuals with cortical EWAS data available. As multiple cortical tissues were available for some cohorts, a mixed-effects model was utilized. In this analysis we controlled for age, sex and neuron/glia proportion, with surrogate variables added as appropriate to reduce inflation. Using this approach, we identified 220 Bonferroni significant probes, of which 168 were annotated to 121 genes, with 52 DMPs unannotated using Illumina UCSC annotation (Fig. 2a, b, Table 2, Supplementary Data 7, Supplementary Fig. 15). All of the 220 probes were nominally significant $(P<0.05)$ in $\geq$ two cohorts, with ten of these probes being nominally significant in all six cohorts (Supplementary Fig. 16), which included single probes annotated to $A N K 1, A B R, S P G 7$ and WDR81, two probes in DUSP27, three probes in $R H B D F 2$ and one unannotated probe. We observed similar DNA methylation patterns across all cortical cohorts and tissues for the 220 probes with 219 of the 220 DMPs showing the same direction of effect in at least five cohorts. In total, 154 of the DMPs were hypermethylated, with 66 hypomethylated, representing an enrichment for hypermethylation $\left(P=4.85 \times 10^{-10}\right)$. This pattern of methylation was evident across all cortical tissues but was not seen in the cerebellum (Supplementary Fig. 17). Of the 220 DMPs we identified, 46 of these have been previously reported at Bonferroni significance in published EWAS, including multiple previously identified probes in ANK1 (cg05066959, cg11 823178), MCF2L (cg07883124, cg09448088), PCNT (cg00621289, cg04147621, cg23449541) and RHBDF2 (cg05810363, cg1216 3800 , cg12309456, cg13076843). The most significant probe we identified in our cross-cortex analysis was cg12307200 (Table 2, $\left.\mathrm{ES}=-0.015, P=4.48 \times 10^{-16}\right)$, which is intergenic and found at chr3:188664632, located between the TPRG1 and LPP genes and had been previously reported at Bonferroni significance by De Jager and colleagues with respect to neuritic plaque burden ${ }^{6}$ and by Brokaw and colleagues with respect to post-mortem diagnosis $^{12}$. Our cross-cortex meta-analysis approach has identified 174 novel DMPs (at Bonferroni significance), annotated to 102 genes. Although 11 of these genes had previously been reported at Bonferroni significance (another probe within that gene), the remaining 96 genes represent robust novel loci in $\mathrm{AD}$. Many of these novel differentially methylated genes had multiple Bonferroni significant probes, for example, five probes in $A G A P 2$, three probes in $H O X B 3$ and $S L C 44 A 2$, and two probes in $C D H 9$, CPEB4, DUSP27, GCNT2, MAMSTR, PTK6, RGMA, RHOB, SMURF1, THBS1, ZNF238 and ZNF385A (Supplementary Data 7). Although some of these loci may have been reported in earlier AD EWAS, none of these were at Bonferroni significance and so here represent robust novel loci.

We were interested to investigate whether specific functional pathways were differentially methylated in $\mathrm{AD}$ cortex and so performed a gene ontology pathway analysis of the 121 genes annotated to the 220 Bonferroni significant cross-cortex DMPs. We highlighted epigenetic dysfunction in numerous pathways (at nominal significance), interestingly including a number of developmental pathways, mainly featuring the HOXA and HOXB gene clusters (Supplementary Data 8). Given that we identified multiple DMPs in some genes, we were interested to investigate the correlation structure between probes in close proximity to each other to establish how many independent signals we had identified. Using a method developed to identify single nucleotide polymorphisms (SNPs) in linkage disequilibrium (LD) ${ }^{14}$, we collapsed the 220 Bonferroni significant loci into 165 independent (non-highly correlated $[r<0.6$ over $1 \mathrm{mb}]$ ) signals (Supplementary Data 9). We found that the largest reduction in signals occurred in the HOXA and HOXB gene clusters, with the 18 DMPs in the HOXA region representing only two independent signals, whilst the seven DMPs in the HOXB region represented one independent signal. Next we undertook a formal regional analysis to identify genomic regions of multiple adjacent DMPs and identified 221 DMRs, with the top DMR containing 11 probes and covering the HOXA region (chr7:27,153,212-27, 154,305: $P=3.84 \times 10^{-35}$ ) (Fig. 2c, Supplementary Data 10). The HOXA gene cluster further featured a number of times in our DMR analysis; four of the ten most significant DMRs fell in this genomic region, including DMRs spanning four probes (chr7:27 146237-27146445: $P=4.11 \times 10^{-27}$ ), 33 probes (chr7:271831 33-27184667: $\left.P=2.22 \times 10^{-20}\right)$ and ten probes (chr7:271432 35-27143806: $P=1.75 \times 10^{-18}$ ).

Replication of pathology associated DMPs in the cortex. To replicate our findings and to determine the cellular origin of DNA methylomic differences we used the estimated coefficients and SEs 


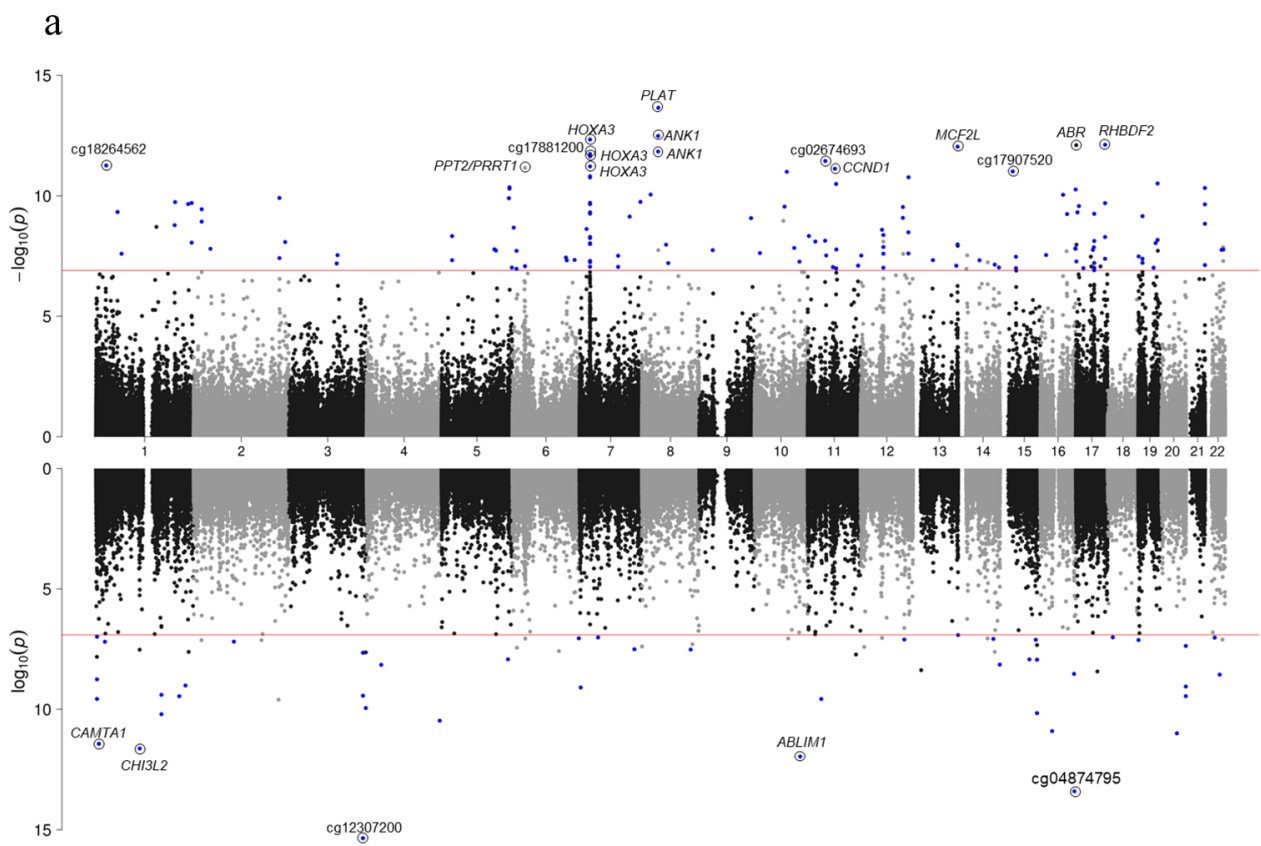

$\mathrm{b}$

c
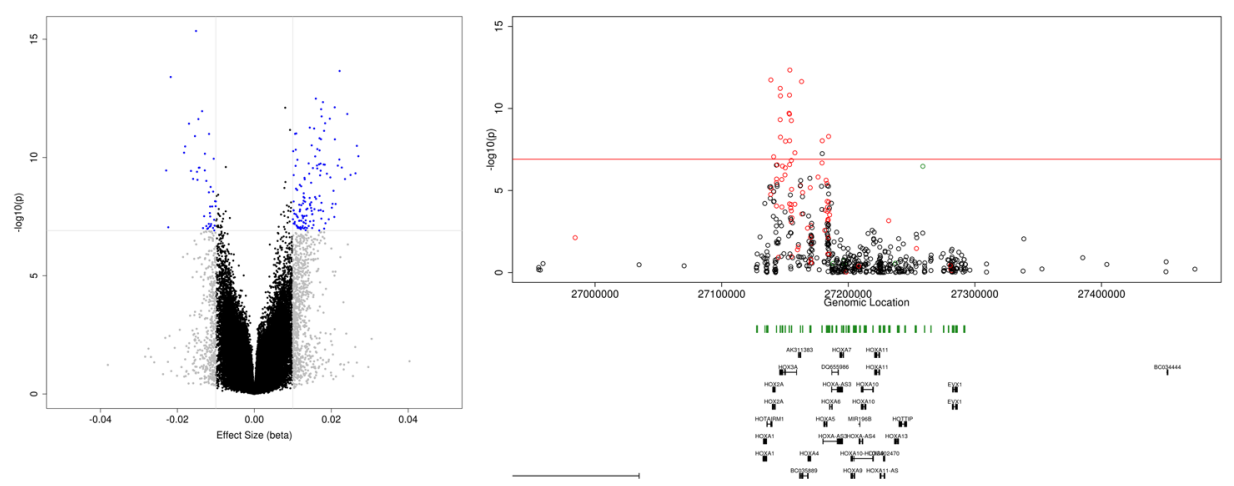

Fig. 2 A cross-cortex meta-analysis identifies 220 Bonferroni significant differentially methylated positions (DMPs) associated with Braak stage. a A Miami plot of the cross-cortex meta-analyses $(N=1408)$. Probes shown above the $X$-axis indicate hypermethylation with higher Braak stage, whilst probes shown below the $X$-axis indicate hypomethylation with higher Braak stage. The chromosome and genomic position are shown on the $X$-axis. The $Y$-axis shows $-\log 10(p)$. The red horizontal lines indicate the Bonferroni significance level of $P<1.238 \times 10^{-7}$. Probes with a methylation (beta) effect size (ES: difference between Braak 0 - Braak VI) $\geq 0.01$ and $P<1.238 \times 10^{-7}$ are shown in blue. The 20 most significant DMPs are circled on the plot and Illumina UCSC gene name is shown if annotated, or CpG ID if unannotated. Exact $P$ values can be found in Table 2 and Supplementary Data 7. b A volcano plot showing the ES $(X$-axis) and $-\log 10(p)(Y$-axis) for the cross-cortical meta-analysis results. Grey probes indicate an ES between $\geq 0.01$, whilst blue probes indicate an $E S \geq 0.01$ and $P<1.238 \times 10^{-7}$. c The most significant cross-cortex differentially methylated region (DMR) (chr7:27153212-27154305) contained 11 probes and resided in the HOXA region. The horizontal red line denotes the Bonferroni significance level of $P<1.238 \times 10^{-7}$. Red probes represent a positive $E S \geq 0.01$, blue probes represent a negative $E S \geq 0.01$. Underneath the gene tracks are shown in black with $C p G$ islands in green.

for these 220 probes generated in a seventh independent (Munich) cohort, which consisted of $450 \mathrm{~K}$ data generated in the prefrontal cortex $(N=45)$ and sorted neuronal and non-neuronal nuclei from the occipital cortex $(N=26)$ (Table 1$)$. This cohort had not been used in our discovery analyses as $<50$ samples were available. Notably, we identified a similar pattern of Braak-associated DNA methylation changes for the 220 Bonferroni significant crosscortex probes in this replication cohort, with a significantly correlated effect size between the discovery dataset and the replication prefrontal cortex $\left(r=0.64, P=5.24 \times 10^{-27}\right)$, neuronal $(r=0.45$, $\left.P=1.56 \times 10^{-12}\right)$ and non-neuronal datasets $(r=0.79, P=1.43 \times$ $10^{-47}$ ) with a similar enrichment for the same direction of effect (sign test: prefrontal cortex $P=4.59 \times 10^{-28}$, neuronal $P=6.13 \times$ $10^{-15}$, non-neuronal $P=1.06 \times 10^{-42}$ ) (Fig. 3a). The most significant probe from the cross-cortex meta-analysis (cg12307200) showed consistent hypomethylation in disease in all cohorts in all cortical brain regions, with this direction of effect replicated in the prefrontal cortex and non-neuronal nuclei samples, but not the neuronal nuclei samples, suggesting that this is primarily driven by non-neuronal cell types, which are likely to be glia (Fig. 3b). We have developed an online database (www.epigenomicslab.com/admeta-analysis/), which can generate a forest plot showing the ES and SE across any of the discovery cohorts and the Munich sample types for any of the 403,763 probes that passed our quality control. This allows researchers to determine the consistency of effects across cohorts for a given CpG site as well as the likely cellular origin of the signature. In addition, our tool can generate miniManhattan plots to show DMRs utilizing the summary statistics from the cross-cortex meta-analysis.

Finally, we had access to DNA methylation data generated in an eighth independent (Brains for Dementia Research [BDR]) cohort. This consisted of Illumina Infinium HumanMethylation EPIC 


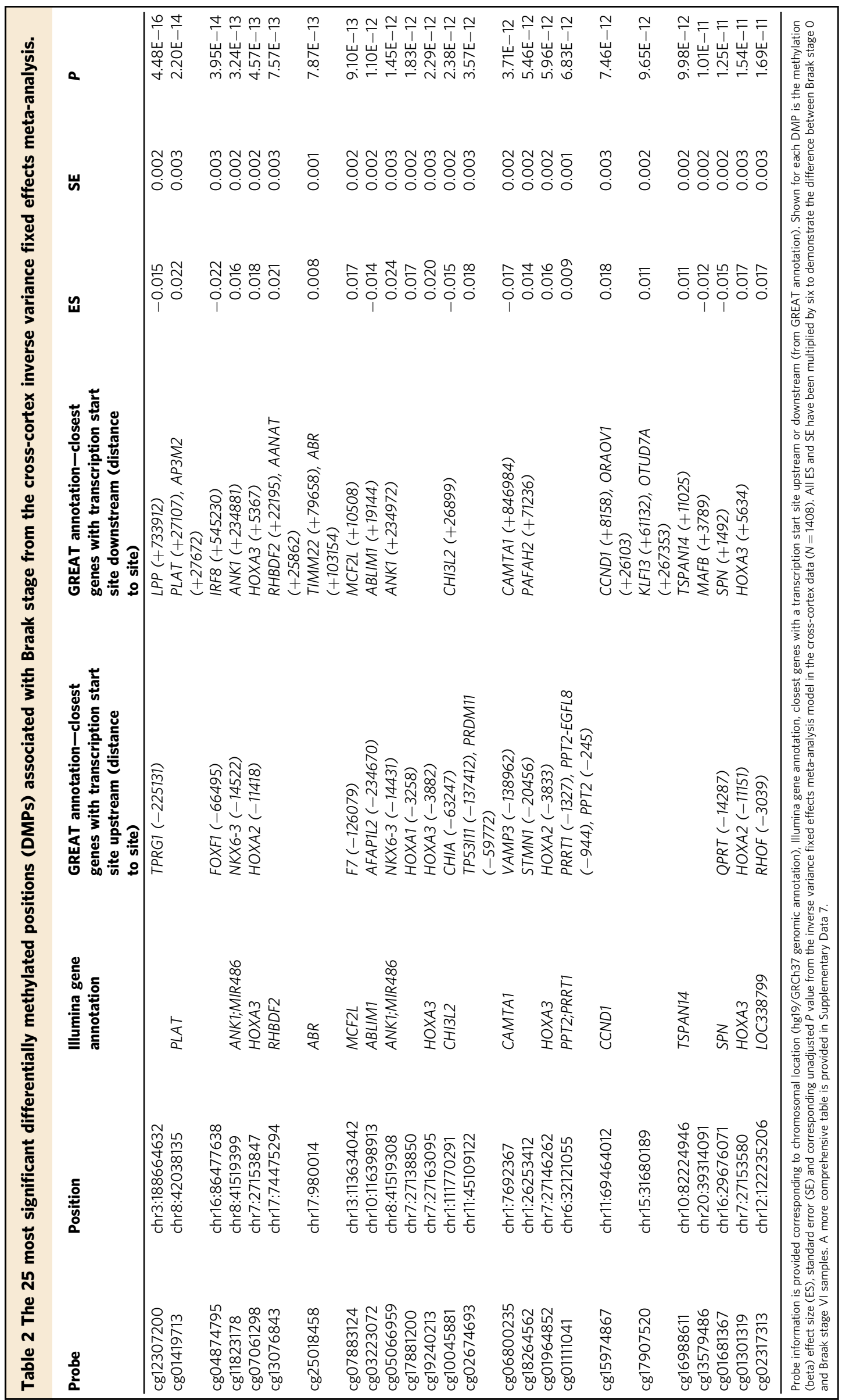


a

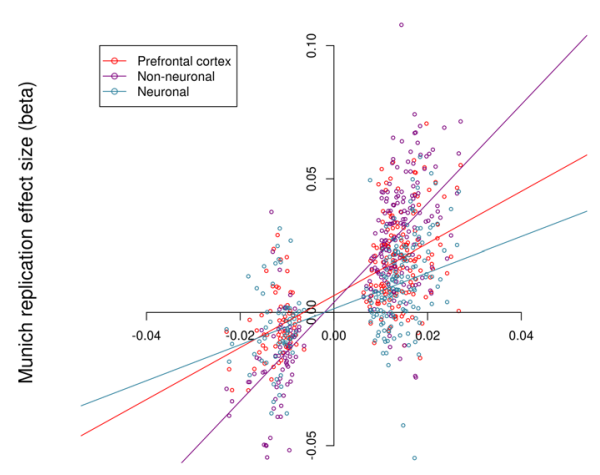

Cross-Cortex effect size (beta) b

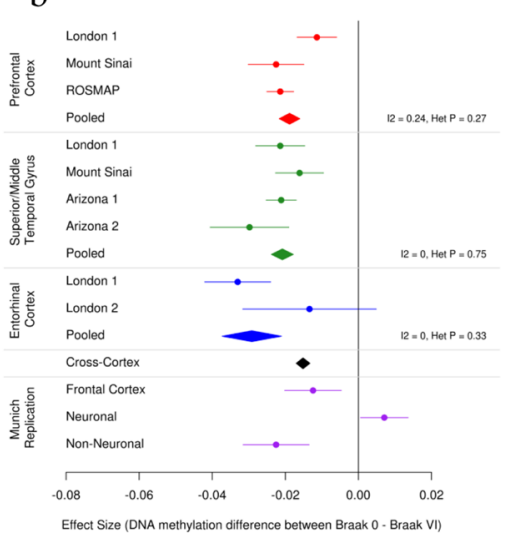

$\mathrm{c}$

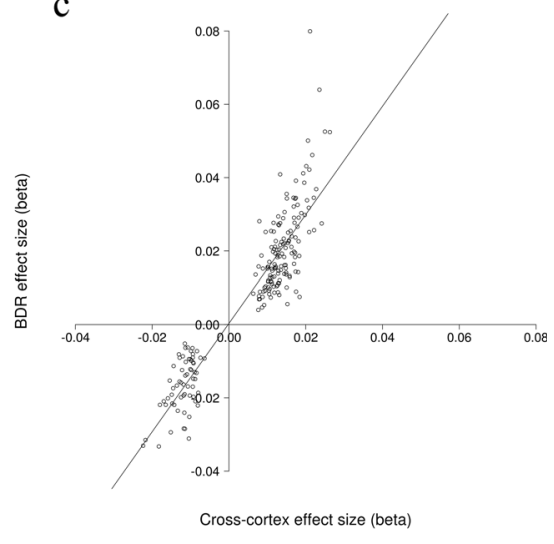

Fig. 3 Independent replication of the Bonferroni significant cross-cortex differentially methylated loci. a The methylation (beta) effect size (ES) of the 220 cross-cortex differentially methylated positions (DMPs) identified in the discovery $(N=1408)$ cohorts $(X$-axis) were significantly correlated with the ES in the Munich replication cohort in the prefrontal cortex (red; $N=45, r=0.64, P=5.24 \times 10^{-27}$ ), sorted neuronal cells (light blue; $N=26, r=0.45, P$ $=1.56 \times 10^{-12}$ ) and non-neuronal cells (purple; $r=0.79, N=26, P=1.43 \times 10^{-47}$ ) ( $Y$-axis). $\mathbf{b}$ A forest plot of the most significant cross-cortex DMP (cg12307200, chr3:188664632, $\left.P=4.48 \times 10^{-16}\right)$. The effect size is shown in the prefrontal cortex (red; $\left.N=961\right)$, temporal gyrus (green; $\left.N=608\right)$ and entorhinal cortex (blue; $N=189$ ) for the different discovery cohorts. The $X$-axis shows the beta $E S$, with dots representing ES and arms indicating standard error (SE). ES from the intra-tissue meta-analysis using all available individual cohorts are represented by polygons in the corresponding tissue colour. The black polygon represents the cross-cortex data. Shown in purple on the plot is the ES in the Munich replication cohort in the prefrontal cortex and sorted neuronal cells and non-neuronal cells, with the direction of effect suggesting the hypomethylation seen in the discovery cohorts is driven by changes in non-neuronal cells. $\mathbf{c}$ In the BDR replication cohort $(N=590)$ DNA methylation data were available in the prefrontal cortex for 208 of the 220 Bonferroni significant cross-cortex DMPs. The ES of these 208 cross-cortex DMPs in the discovery cohorts ( $X$-axis) were significantly correlated with the ES in the BDR replication cohort $\left(r=0.53, P=4.13 \times 10^{-16}\right)(Y$-axis $)$.

BeadChip (EPIC array) data in the prefrontal cortex in 590 individuals ${ }^{15}$. As this is the successor to the $450 \mathrm{~K}$ array (which had been used for the other seven cohorts), there are some differences in genome coverage, and for the 220 Bonferroni significant cross-cortex DMPs we had identified in the discovery cohorts, only 208 probes are also present on the EPIC array. For these overlapping 208 probes, we observed a significantly correlated effect size between the discovery dataset and the BDR dataset $\left(r=0.53, P=4.13 \times 10^{-16}\right)$ (Fig. 3c), with all 208 probes showing the same direction of effect (sign test $P=4.86 \times 10^{-63}$ ).

Cross-cortex AD-associated DMPs are enriched in specific genomic features. To identify if the cross-cortex DMPs reside in specific genomic features, we used a Fisher's exact test to look for an enrichment of the 220 DMPs using Slieker annotations ${ }^{16}$ (Supplementary Data 11, Supplementary Fig. 18). We observed a significant over-representation of Bonferroni significant DMPs in $\mathrm{CpG}$ islands of gene bodies (odds ratio $[\mathrm{OR}]=3.199, P=4.76 \times$ $10^{-10}$ ), and in $\mathrm{CpG}$ island shelves and non-CpG island areas of proximal promoters $\left(\mathrm{OR}=3.571, P=9.09 \times 10^{-3}\right.$ and $\mathrm{OR}=$ 1.641, $P=0.03$, respectively). However, DMPs located in CpG islands in the proximal promoter were under-represented $(\mathrm{OR}=$ $\left.0.353, \quad P=2.08 \times 10^{-6}\right)$. There was a significant overrepresentation of the 220 cross-cortex DMPs in the first exon $(\mathrm{OR}=1.80, P=0.02)$, with an under enrichment within $1500 \mathrm{bp}$ of the transcription start site $\left(\mathrm{OR}=0.49, \quad P=3.82 \times 10^{-3}\right)$ (Supplementary Data 12, Supplementary Fig. 19).

DNA methylomic signatures in the cortex can explain variance in the degree of pathology. We were interested to investigate whether the Braak-associated DNA methylation patterns we had identified across the cortex could accurately predict the pathological load of a brain sample and how much variance this explained. To this end, we took samples within the discovery cohorts with either low pathology (Braak 0-II [controls]: $N=$
407), or high pathology (Braak V-VI $[\mathrm{AD}]: N=589$ ) and used these as a training dataset. We then used elastic net regression to identify 110 probes in the 220 cross-cortex Bonferroni significant loci (Supplementary Data 13) that were able to explain the most variance between post-mortem low pathology [control] from high pathology $[\mathrm{AD}]$ status in our training dataset $(N=996)$ (Supplementary Data 14, Fig. 4). In our training data, we achieved an area under the curve (AUC) of the receiver operating characteristic $(\mathrm{ROC})$ of $94.33 \%(\mathrm{CI}=92.88-95.64 \%$, variance explained $=$ $71.11 \%)$. We then tested its performance in the Munich replication samples $(N=38)$ and the BDR replication samples $(N=$ 454), where it achieved an AUC of $73.95 \%$ ( CI $=55.17-88.89 \%$, variance explained $=20.18 \%)$ and $70.36 \%(C I=65.52-75.12 \%$, variance explained $=15.87 \%$ ), respectively (Supplementary Data 14, Fig. 4).

DNA methylation signatures in AD cortex are largely independent of genetic effects. DNA methylomic variation can be driven by genetic variation via methylation quantitative trait loci (mQTLs). To explore whether SNPs may be driving the methylation differences we observed (in cis) we used the XQTL resource to identify cis-mQTLs associated with the 220 Bonferroni significant cross-cortex DMPs ${ }^{17}$. We identified 200 Bonferroni corrected mQTLs, which were associated with DNA methylation at 18 of the 220 cross-cortex DMPs (Supplementary Data 15). This suggests that the majority of Braak-associated DMPs are not the result of genetic variation in cis. None of these mQTLs overlapped with lead SNPs (or SNPs in LD) identified in the most recent genome-wide association study (GWAS) of diagnosed lateonset $\mathrm{AD}$ from Kunkle et al. $^{18}$. Next, we were interested in exploring whether DNA methylation is enriched in genes known to harbor $\mathrm{AD}$-associated genomic risk variants. Using the $\mathrm{AD}$ variants from Kunkle et al. ${ }^{18}$ we examined the enrichment of Braak-associated DNA methylation in 24 LD blocks harbouring risk variants. Twenty of these LD blocks contained $>1 \mathrm{CpG}$ site on the $450 \mathrm{~K}$ array and using Brown's method we combined $P$ 


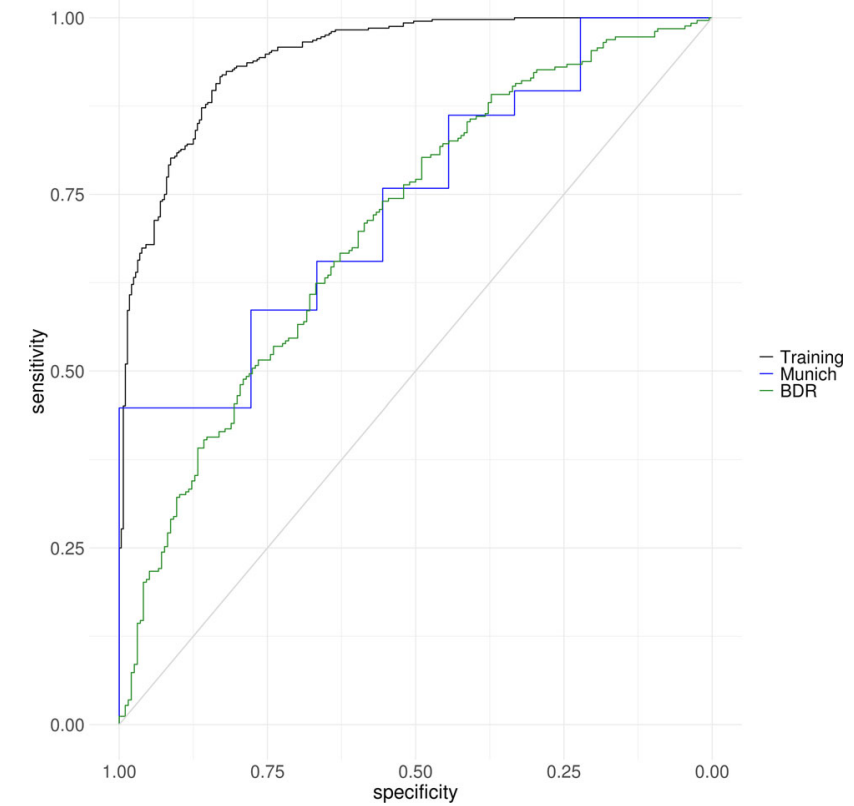

Fig. 4 Receiver operating characteristic (ROC) graphs highlighting the area under the curve (AUC) for the $\mathbf{1 1 0}$ cross-cortex probes that can best explain the variance in Braak pathology. An elastic net penalized

regression model was used to identify a subset of 110 of the Bonferroni significant cross-cortex probes that could best predict whether a sample has low pathology (Braak 0-II: control) compared to high pathology (Braak $\mathrm{V}-\mathrm{VI}: \mathrm{AD}$ ) in a training dataset comprised of 996 discovery samples (Braak $0-I I: N=407$, Braak V-VI: $N=589$ ). This model had an area under the ROC curve (AUC) of $94.33 \%$ (confidence interval $[\mathrm{Cl}]=92.88-95.64 \%$ ) and explained $71.11 \%$ of the pathological variance (black line). The 110 probe signature was then tested in two independent replication cohorts. In the Munich prefrontal cortex samples (Braak $0-I I: N=9$, Braak V-VI: $N=$ 29) the model had an AUC of $73.95 \%(C l=55.17-88.89 \%)$, explaining $20.18 \%$ of the variance (blue line). In the BDR prefrontal cortex samples (Braak 0-II: $N=196$, Braak V-VI: $N=258$ ) the model had an AUC = $70.36 \%(\mathrm{Cl}=65.52-75.12 \%)$, explaining $15.87 \%$ of the variance (green line). A list of the 110 probes and their performance characteristics can be found in Supplementary Data 13 and 14, respectively.

values within each of these 20 genomic regions. We observed Bonferroni-adjusted significant enrichment in the cross-cortex data in the HLADRB1 (Chr6: 32395036-32636434: adjusted $P=$ $1.20 \times 10^{-3}$ ), SPI1 (Chr11: 47372377-47466790, adjusted $P=$ $\left.5.76 \times 10^{-3}\right)$, SORL1 (Chr11: 121433926-121461593, adjusted $P=0.019$ ), ABCA7 (Chr19: 1050130-1075979, adjusted $P=$ 0.022 ) and $A D A M 10$ (Chr15: 58873555-59120077, adjusted $P=$ 0.022) LD regions (Supplementary Data 16).

\section{Discussion}

This meta-analysis of AD EWAS utilizes six published independent sample cohorts with a range of cortical brain regions and cerebellum available as a discovery dataset. Two further independent cortical datasets were then used for replication, including data from sorted nuclei populations. Our data can be explored as part of an online searchable database, which can be found on our website (https://www.epigenomicslab.com/ad-meta-analysis). By performing a meta-analysis within each tissue, we have been able to identify 236, 95 and ten Bonferroni significant DMPs in the prefrontal cortex, temporal gyrus and entorhinal cortex, respectively. Although far fewer loci were identified in the entorhinal cortex compared to the other cortical regions, this is likely due to the reduced sample size in this tissue. In the cerebellum despite meta-analyzing $>500$ unique samples, we identified no Braakassociated DNA methylation changes. Furthermore, there was no correlation of the effect size of Bonferroni significant DMPs identified in any of the cortical regions with the effect size of the same probes in the cerebellum. Taken together, this suggests that DNA methylomic changes in $\mathrm{AD}$ are cortex cell type specific. This observation is interesting as the cerebellum is said to be spared from $\mathrm{AD}$ pathology, with an absence of neurofibrillary tangles, although some diffuse amyloid-beta plaques are reported ${ }^{19}$. Interestingly, a recent spatial proteomics study of $\mathrm{AD}$ reported a large number of protein changes in the cerebellum in $\mathrm{AD}$; however, the proteins identified were distinct from other regions examined and thus the authors suggested a potential protective role ${ }^{20}$.

Although many loci showed similar patterns of Braakassociated DNA methylation across the different cortical regions, some loci did show some regional specificity. In order to identify $\mathrm{CpG}$ sites that showed common DNA methylation changes in disease we performed a cross-cortex meta-analysis. Using this approach we identified 220 Bonferroni significant probes associated with Braak stage of which 46 probes had been previously reported at Bonferroni significance in the individual cohort studies that we had used for our meta-analysis, for example, two probes in $A N K 1$, four probes in $R H B D F 2$ and one probe in $H O X A 3$, amongst others. Interestingly, our approach did identify 174 novel CpGs, corresponding to 102 unique genes, of which 84 genes had not been previously reported at Bonferroni significance in any of the previously published AD brain EWAS, highlighting the power of our meta-analysis approach for nominating new loci. This included 15 novel genes with at least two Bonferroni significant DMPs each, including five probes in $A G A P 2$, three probes in SLC44A2 and two probes each in $C D H 9$, CPEB4, DUSP27, GCNT2, MAMSTR, PTK6, RGMA, RHOB, SMURF1, THBS1, ZNF238 and ZNF385A. These genes had not been identified previously in an AD EWAS at this significance threshold, although a number of these genes had been previously identified from DMR analyses, which have a less stringent threshold. However, we did identify one novel gene (HOXB3) with three Bonferroni significant DMPs, which had not been identified at this significance threshold in previous EWAS DMP or DMR analyses in $\mathrm{AD}$ brain. The nomination of loci in the $H O X B$ gene cluster is interesting; a recent study of human Huntington's disease brain samples also highlighted significantly increased $H O X B 3$ gene expression in the prefrontal cortex ${ }^{21}$, an interesting observation given that both $\mathrm{AD}$ and Huntington's disease are disorders that feature dementia. Furthermore, we have recently reported $\mathrm{AD}$-associated hypermethylation of the HOXB6 gene in AD blood samples ${ }^{22}$. Our pathway analysis highlighted differential methylation in a number of developmental pathways, mainly featuring the HOXA and $H O X B$ gene clusters. Although it is unclear why developmental genes may be changed in a disease that primarily affects the elderly, it has been implied that genes such as these may be involved in neuroprotection after development ${ }^{23}$. A number of the other novel genes with multiple DMPs are also biologically relevant in the context of $\mathrm{AD}$, for example, GCNT2 was recently shown to be differentially expressed in the Putamen between males and females with $\mathrm{AD}^{24}$. Interestingly, some of the protein products of genes we identified have also been previously linked with AD; PTK6 is a protein kinase whose activity has been shown to be altered in postmortem AD brain ${ }^{25}$. Similarly, RGMA has been shown to be increased in $\mathrm{AD}$ brain, where it accumulated in amyloid-beta plaques $^{26}$.

Our genomic enrichment analyses identified an overrepresentation of hypermethylated loci in $\mathrm{AD}$ and methylation 
in specific genomic features, for example, $\mathrm{CpG}$ islands in gene bodies, and shelves and non-CpG island regions in proximal promoters. We demonstrated that the majority of DMPs we identified $(N=202)$ were not driven by genetic variation as only 18 of the $220 \mathrm{CpG}$ sites have reported mQTLs. However, we did observe a significant enrichment of cross-cortex loci in the LD regions surrounding the $\mathrm{AD}$-associated genetic variants HLADRB1, SPI1, SORL1, ABCA7 and ADAM10 after controlling for multiple testing. Finally, we have developed a classifier that could accurately predict control samples with low pathology, from those with a post-mortem $\mathrm{AD}$ diagnosis due to high pathology using methylation values for 110 of the 220 Bonferroni significant probes, further highlighting that distinct genomic loci reproducibly show epigenetic dysfunction in $\mathrm{AD}$ cortex. Although the clinical utility of such a classifier is limited as it is developed in post-mortem cortical brain tissue, it does illustrate that specific robust patterns of DNA methylation differences occur as the disease progresses. These signatures require further investigation as they could represent novel therapeutic targets, particularly given the classifier had an AUC $>70 \%$ in all the training and replication datasets. However, it is worth noting that the variance explained by the $110 \mathrm{CpG}$ signature was lower in the replication datasets than the discovery samples, which could be due to a low sample number (Munich) or the different Illumina array platform (BDR).

There are some limitations with our study. First, as we have largely utilized methylation data generated in bulk tissue, this will contain a mixture of different cell types. Furthermore, it is known that the proportions of the major brain cell types are altered in $\mathrm{AD}$, with reduced numbers of neurons and increased glia. As such, it is possible that the identified DNA methylation changes represent a change in cell proportions. To address this, we have included neuron/glia proportions as a co-variate in our models to minimize bias and used data from sorted cell populations as part of our replication. Although this is the optimal strategy for the current study given the EWAS data had already been generated, future EWAS should be undertaken on sorted cell populations with larger sample numbers than the Munich replication cohort, or ideally at the level of the single cell. It is important to note that the data from the sorted nuclei populations in the Munich replication cohort were generated in the occipital cortex, which was not a bulk tissue used for any of the discovery cohorts. In the future it would be interesting to explore whether different diseaseassociated DNA methylation signatures were observed in neurons and glia isolated from different cortical brain regions. Second, our study has utilized previously generated EWAS data generated on the $450 \mathrm{~K}$ array or EPIC array. Although the Illumina array platform has been the most widely used platform for EWAS to date, it is limited to only analyzing a relatively small proportion of the potential methylation sites in the genome $(\sim 400,000$ on the $450 \mathrm{~K}$ array) and given the falling cost of sequencing, future studies could exploit this by performing reduced representation bisulfite sequencing to substantially increase the coverage. In our study we have primarily used the UCSC annotation provided by Illumina to identify the gene relating to each DMP. However, this can lead to the annotation of overlapping genes, or no gene annotation, which can make it difficult to establish the gene of interest in the absence of functional studies. Our study has primarily focused on the results of a fixed-effects meta-analysis, as the majority of Bonferroni significant DMPs do not display a high degree of heterogeneity. However, $\sim 15 \%$ of the cross-cortex DMPs did have a significant heterogeneity $P$ value and in this instance, it is worthwhile also considering the results of the random-effects meta-analysis. Although this heterogeneity could be driven by differences between cohorts, it is also plausible that it may be driven by tissue-specific effects as we used different cortical brain regions in the model. For example, cg22962123 annotated to the HOXA3 gene has a significant heterogeneity $P$ value in the cross-cortex meta-analysis, but we had already shown this loci to be differentially methylated in the prefrontal cortex and temporal gyrus, but not the entorhinal cortex in our intratissue meta-analysis.

Another limitation of our study is that we have focused our analyses on Braak (neurofibrillary tangle)-associated methylation changes, as this measure was available in all cohorts. Given that amyloid-beta is another neuropathological hallmark of $\mathrm{AD}$, it would also be of interest to identify neuritic plaque-associated DMPs. Unfortunately, this was not feasible in the current study as this measure was not available in all samples. In a similar vein, we did not exclude individuals with mixed pathology, or protein hallmarks of other neurodegenerative diseases, such as the presence of lewy bodies, or TDP-43 pathology. In the future, larger meta-analyses should stratify by the presence of these protein aggregates, particularly given that very few EWAS have been undertaken in other dementias. Indeed, only three DNA methylomic studies have been undertaken in cortical samples of individuals with other dementias to date ${ }^{27-30}$, with none of these studies utilizing $>15$ individuals for EWAS. Further studies exploring common and unique DNA methylation signatures and our classifier in other diseases characterized by dementia will be vital for identifying disease-specific epigenetic signatures that could represent novel therapeutic targets. Finally, one key issue for epigenetic studies in post-mortem tissue is the issue of causality, where it is not possible to determine whether diseaseassociated epigenetic loci are driving disease pathogenesis, or are a consequence of the disease, or even the medication used for treatment. One method that can be used to address this is Mendelian Randomization ${ }^{31}$ however, this does require the CpG site to have a strong association with a SNP. Given that we only identified mQTLs at 18 of the 220 Bonferroni significant crosscortex DMPs, this approach is not suitable for most of the loci we identified. At an experimental level establishing causality is difficult to address in post-mortem human studies, and therefore longitudinal studies in animal models, or modelling methylomic dysfunction through epigenetic editing in vitro will be useful approaches to address these issues. In addition, examining DNA methylation signatures in brain samples in pre-clinical individuals (i.e. during midlife) will be important for establishing the temporal pattern of epigenetic changes relative to the pathology.

In summary, we present intra-tissue and cross-cortex metaanalyses of AD EWAS, highlighting numerous Bonferroni significant DMPs in the individual cortical regions and across the cortex, but not in the cerebellum, which were replicated in two independent cohorts. A number of these loci are novel and warrant further study to explore their role in disease etiology. We highlight that the nominated epigenetic changes are largely independent of genetic effects, with only 18 of the 220 Bonferroni significant DMPs showing a mQTL. We provide evidence that robust epigenomic changes in the cortex can predict the level of pathology in a sample. Looking to the future it will be important to explore the relationship between DNA methylation and gene expression in $\mathrm{AD}$ brain.

\section{Methods}

Cohorts. Six sample cohorts were used for discovery in this study as they all had DNA methylation data generated on the $450 \mathrm{~K}$ array for $>50$ donors, enabling us to take a powerful meta-analysis approach to identify DNA methylation differences in $\mathrm{AD}$. As our analyses focused specifically on neuropathology (tau)-associated differential methylation, inclusion criteria for all samples used in the discovery or replication cohorts was having post-mortem neurofibrillary tangle Braak stage available. For each discovery sample cohort DNA methylation was quantified using the $450 \mathrm{~K}$ array. The London 1 cohort comprised of prefrontal cortex, superior temporal gyrus, entorhinal cortex, and cerebellum tissue obtained from 113 
individuals archived in the MRC London Neurodegenerative Disease Brain Bank and published by Lunnon et al. ${ }^{5}$. The London 2 cohort comprised entorhinal cortex and cerebellum samples obtained from an additional 95 individuals from the MRC London Neurodegenerative Disease Brain Bank published by Smith and colleagues $^{8}$. The Mount Sinai cohort comprised of prefrontal cortex and superior temporal gyrus tissue obtained from 146 individuals archived in the Mount Sinai Alzheimer's Disease and Schizophrenia Brain Bank published by Smith and colleagues $^{7}$. The Arizona 1 cohort consisted of 302 middle temporal gyrus and cerebellum samples from The Sun Health Research Institute Brain Donation Program ${ }^{32}$ published by Brokaw et al. ${ }^{12}$. The Arizona 2 cohort consisted of an additional 88 temporal gyrus and cerebellum samples from Lardonije et al. ${ }^{10}$. The ROSMAP cohort consisted of 711 samples from the Rush University Medical Center: Religious Order Study (ROS) and the Memory and Aging Project (MAP), which were previously published by De Jager and colleagues ${ }^{6}$. For replication purposes we used two further replication datasets. The Munich cohort from Neurobiobank Munich (NBM), which had bulk prefrontal cortex $450 \mathrm{~K}$ array data from 45 donors, and $450 \mathrm{~K}$ array data from fluorescence-activated cell sorted neuronal and non-neuronal (glial) populations from the occipital cortex from 26 donors as described by Gasparoni et al. ${ }^{11}$. The Brains for Dementia Research (BDR) cohort consisted of bulk prefrontal cortex Illumina Infinium EPIC array data from 590 donors, as described by Shireby et al. ${ }^{15}$. Demographic information for all eight cohorts is available in Table 1. Ethical approval for the study was granted from the University of Exeter Medical School Research Ethics Committee $(13 / 02 / 009)$

Data quality control and harmonization. All computations and statistical analyses were performed using R $3.5 .2^{33}$ and Bioconductor $3.8^{34}$. A MethylumiSet object was created from iDATs using the readEPIC function in the wateRmelon package ${ }^{35}$ and RGChannelSet object was created using the minfi package ${ }^{35}$. Samples were excluded from further steps if (a) the mean background intensity of negative probes $<1000$, (b) the mean detection $P$ values $>0.005$, (c) the mean intensity of methylated or unmethylated signals were three standard deviations above or below the mean, (d) the bisulfite conversion efficiency $<80 \%$, (e) there was a mismatch between reported and predicted sex, or (f) the $65 \mathrm{SNP}$ probes on the array show a modest level of correlation (using a cut-off of 0.65 ) between two samples (whereby the sample with the higher Braak score was retained). Sample and probe exclusion was performed using the pfilter function within the wateRmelon package ${ }^{36}$, with the following criteria used for exclusion: samples with a detection $P>0.05$ in more than $5 \%$ of probes, probes with $<$ three beadcount in $5 \%$ of samples and probes having $1 \%$ of samples with a detection $P$ value $>0.05$. Finally, probes with common (minor allele frequency $>5 \%$ ) SNPs in the single base extension position or probes that are nonspecific or mis-mapped were excluded $^{37,38}$, leaving 403,763 probes for analysis. Samples numbers after quality control are those shown in Table 1.

Quantile normalization was applied using the dasen function in the wateRmelon package ${ }^{36}$. For the discovery cohorts, DNA methylation data were corrected by regressing out the effects of age and sex in all samples in each cohort and tissue separately, with neuron/glia proportions included as an additional covariate in cortical regions. The neuron/glia proportions were calculated using the CETS package ${ }^{39}$, and were not included as a co-variate for the cerebellum as the neuronal nuclear protein $(\mathrm{NeuN})$ that was used to generate the neuron/glia algorithm is not expressed by some cerebellar neurons ${ }^{40}$. These three variables (age, sex, neuron/glia proportions) were regressed out of the data as we found that they strongly correlated with either of the first two principal components of the DNA methylation data in most of the datasets. Other potential sources of technical and biological variation (post-mortem interval, ancestry, plate, chip, study and bisulfite treatment batch) did not correlate as strongly with methylation in most datasets. We opted to use surrogate variables as a consistent method to control for variation derived from these measured and other unknown variables across all datasets. Surrogate variables were calculated using the sva function in the SVA package ${ }^{41}$. Linear regression analyses were then performed with respect to Braak stage (modelled as a continuous variable) using residuals and a variable number of surrogate variables for each study until the inflation index (lambda) fell below 1.2 (see Supplementary Data 17). The surrogate variables included for each cohort correlated with the technical and biological variables that we had not regressed out earlier, demonstrating that this method appropriately controlled for variation not driven by Braak stage. Quantile-quantile plots for the four intra-tissue and the cross-cortex meta-analyses are shown in Supplementary Fig. 20. Although it appears from these plots that there is $P$ value inflation, it is worth noting that (a) lambda for all meta-analyses $<1.2$ and (b) $P$ value inflation is commonly observed in many DNA methylation studies and standard methods to control for this in GWAS are not suitable for EWAS data ${ }^{42}$.

Intra-tissue meta-analysis. We used the estimated coefficients and SEs from the six discovery cohorts to undertake an inverse variance intra-tissue meta-analysis independently in each available tissue using the metagen function within the Meta package ${ }^{43}$, which applies inverse variance weighting. The estimates and SEs from individual cohort Braak linear regression analyses were added to the model for each tissue. The prefrontal cortex analyses included three cohorts $(N=961$ : London 1 , Mount Sinai, ROSMAP), the temporal gyrus analyses included four cohorts $(N=$
608: London 1, Mount Sinai, Arizona 1, Arizona 2) and the entorhinal cortex analyses included two cohorts $(N=189$ : London 1 , London 2$)$. The cerebellum analyses included data from four cohorts $(N=533$ : London 1 , London 2 , Arizona 1 and Arizona 2) although the cerebellum data for the Arizona 1 and 2 cohorts was generated in the same experiment, and so these were combined together as a single dataset. The ESs and corresponding SEs reported in this study correspond to the corrected DNA methylation (beta) difference between Braak 0 and Braak VI individuals. Bonferroni significance was defined as $P<1.238 \times 10^{-7}$ to account for 403,763 tests. A fixed effects meta-analysis are the results primarily reported as it is the most appropriate model for our study as it can more reliably estimate the pooled effect and therefore the standard error and $P$ value. However, in the Supplementary Data we do also report the results of the random-effects meta-analysis as $\sim 10 \%$ of Bonferroni significant DMPs in the intra-tissue meta-analysis had high heterogeneity and in which case the results from the random-effects model should also be considered.

Cross-cortex meta-analysis. As multiple cortical brain regions were available for the London 1 and Mount Sinai cohorts, a mixed model was performed using the lme function within the nlme package ${ }^{44}$. Estimate coefficients and SEs from each EWAS were extracted and were subjected to bacon ${ }^{42}$ to control for bias and inflation, after which a fixed-effect inverse variance meta-analysis was performed across all discovery cohorts using the metagen function. A fixed effects model was selected in this instance for consistency with the intra-tissue meta-analysis, although the random-effects meta-analysis results also shown in Supplementary Data 7.

Replication analyses. For the Munich replication cohort, we extracted the beta values for the 220 cross-cortex Bonferroni significant DMPs. This DNA methylation data were then corrected for age, sex and neuron/glia proportions (bulk tissue only) prior to performing a linear regression analysis with respect to Braak stage. For the BDR replication cohort, we were provided with beta values for the 208 cross-cortex Bonferroni significant DMPs that were present on the EPIC array. This data had been corrected for age, sex, neuron/glia proportions, batch and principal component 1 , before the linear regression analysis was performed with respect to Braak stage, with Bacon used to control for inflation. Additional information on the BDR dataset can be found in Shireby et al. ${ }^{15}$.

Annotations, pathway and regional analyses. Probes were annotated for tables using both the Illumina (UCSC) gene annotation (which is derived from the genomic overlap of probes with RefSeq genes or up to $1500 \mathrm{bp}$ from the transcription start site of a gene) and Genomic Regions Enrichment of Annotations Tool (GREAT) ${ }^{45}$ annotation version 4.0.4 (which annotates a DMP to genes with a transcription start site within $5 \mathrm{~kb}$ upstream, or $1 \mathrm{~kb}$ downstream). Pathway analyses were performed on the Illumina (UCSC) annotated genes corresponding to the 220 Bonferroni significant cross-cortex DMPs $(N=121$ genes). We used the 'gometh' function within the missMethyl package (version 1.20.0) ${ }^{46}$, which performs one-sided hypergeometric tests and adjusts the test for the uneven number of probes per gene and pathway redundancy. The identified GO terms were subjected to the online tool REViGO (available at http://revigo.irb.hr/) ${ }^{47}$, to reduce the number of redundant functional terms based on semantic similarity between ontology terms. Resnik's measure was used to compute the similarity of terms and a medium between terms similarity of 0.7 was allowed. As methylation at neighboring CpG sites can be highly correlated we used a method developed to identify SNPs in LD to identify independent signals ${ }^{14}$. For the 220 Bonferroni significant cross-cortex DMPs we used a threshold of $r<0.6$ over $1 \mathrm{mb}$ to identify 165 independent (nonhighly correlated) methylation signals. To identify DMRs consisting of multiple DMPs we used the Python package comb- $\mathrm{p}^{48}$ with a distance of $500 \mathrm{bp}$ and a seeded $P$ value of $1.0 \times 10^{-4}$. Comb-p was selected for DMR identification over alternative methods as it uses $P$ values as input and so was the most suitable method for calling DMRs in the cross-cortex meta-analysis where multiple brain regions were available for some of the individuals. We have used comb-p to call DMRs in a number of our previous EWAS in $\mathrm{AD}$, including studies where we have validated the top DMRs using an alternative technology such as pyrosequencing ${ }^{5,8,22}$.

Genomic enrichment analyses. To test for an enrichment of DMPs in specific genomic features (i.e. CpG islands, shelves, shores, non-CpG island regions) in certain genomic regions (i.e. intergenic, distal promoter, proximal promoter, gene body, downstream) we annotated all DMPs with Slieker annotation ${ }^{16}$ and performed a two-sided Fisher's exact test comparing to all probes analysed $(N=$ 403,763). We also used a Fisher's exact test to test for an enrichment of DMPs in genomic regions related to transcription based on the Illumina annotation (TSS1500, TSS200, $5^{\prime}$ UTR, $1^{\text {st }}$ exon, gene body, $3^{\prime}$ UTR). To investigate whether any of the 220 Bonferroni significant cross-cortex DMPs were driven by genetic variation we used the $\mathrm{xQTL}$ resource to identify which of these DMPs are established cis-mQTLs ${ }^{17}$. To explore whether Braak-associated methylation was enriched in known AD GWAS variants we used Brown's method to combine together $P$ values from our meta-analyses for probes residing in the LD blocks around the genome-wide significant $\left(P<5.0 \times 10^{-8}\right)$ GWAS variants identified by the stage one meta-analysis of Kunkle et al. ${ }^{18}$. Of the 24 LD blocks reported by Kunkle and 
colleagues, 20 contained $>1 \mathrm{CpG}$ site on the $450 \mathrm{~K}$ array and the $P$ values for each $\mathrm{CpG}$ in a given block were combined using Brown's method, which accounts for the correlation structure between probes, with the regional $P$ values adjusted to correct for multiple testing.

Quantifying variance in Braak pathology explained by DNA methylation signatures. For this analysis control samples (Braak low [0-II]: $N=407$ ) and $\mathrm{AD}$ cases (Braak high [V-VI]: $N=589$ ) from the cross cortex discovery dataset were used for training a classifier. A penalized regression model was used to select the optimum $(N=110)$ CpG probes from the 220 cross-cortex Bonferroni significant DMPs that determined case-control status in the training dataset using the $\mathrm{R}$ package GLMnet ${ }^{49}$. Elastic net uses a combination of ridge and lasso regression, in which alpha $(\alpha)=0$ corresponds to ridge, whilst $\alpha=1$ corresponds to lasso, the elastic net $\alpha$ parameter used was 0.5 . The lambda value was derived when using 10 fold cross validation on the training dataset. The model was then tested for AUC ROC value, confidence intervals $(\mathrm{CI})$ and variance explained in the testing dataset as well as the independent replication Munich (Braak 0-II: $N=9$, Braak V-VI: $N=29$ ) and BDR (Braak 0-II: $N=196$, Braak V-VI: $N=258$ ) prefrontal cortex datasets.

Reporting summary. Further information on research design is available in the Nature Research Reporting Summary linked to this article.

\section{Data availability}

The data supporting the findings of this study are available within the article, Supplementary Information or from the authors upon request. Some of the datasets are also available on GEO including London 1 data (GSE59685), London 2 data (GSE105109), Mount Sinai data (GSE80970), Arizona 1 data (GSE134379), Arizona 2 data (GSE109627) and Munich data (GSE66351). The BDR data is available from the authors upon reasonable request. We have developed an online database, which can present summary statistics, which is available from our website: www.epigenomicslab. com/ad-meta-analysis/.

\section{Code availability}

All scripts for data analyses performed in this manuscript can be found at: https://github. com/rgs212/Meta-analysis-Smith ${ }^{50}$

Received: 25 February 2020; Accepted: 16 April 2021;

Published online: 10 June 2021

\section{References}

1. Collaborators, G. B. D. D. Global, regional, and national burden of Alzheimer's disease and other dementias, 1990-2016: a systematic analysis for the Global Burden of Disease Study 2016. Lancet Neurol. 18, 88-106 (2019).

2. Blennow, K., de Leon, M. J. \& Zetterberg, H. Alzheimer's disease. Lancet 368, 387-403 (2006).

3. Sperling, R. A. et al. Toward defining the preclinical stages of Alzheimer's disease: recommendations from the National Institute on Aging-Alzheimer's Association workgroups on diagnostic guidelines for Alzheimer's disease. Alzheimers Dement 7, 280-292 (2011).

4. Jack, C. R. Jr. et al. Hypothetical model of dynamic biomarkers of the Alzheimer's pathological cascade. Lancet Neurol. 9, 119-128 (2010).

5. Lunnon, K. et al. Methylomic profiling implicates cortical deregulation of ANK1 in Alzheimer's disease. Nat. Neurosci. 17, 1164-1170 (2014).

6. De Jager, P. L. et al. Alzheimer's disease: early alterations in brain DNA methylation at ANK1, BIN1, RHBDF2 and other loci. Nat. Neurosci. 17, 1156-1163 (2014).

7. Smith, R. G. et al. Elevated DNA methylation across a $48-\mathrm{kb}$ region spanning the HOXA gene cluster is associated with Alzheimer's disease neuropathology. Alzheimers Dement 14, 1580-1588 (2018).

8. Smith, A. R. et al. Parallel profiling of DNA methylation and hydroxymethylation highlights neuropathology-associated epigenetic variation in Alzheimer's disease. Clin. Epigenetics 11, 52 (2019).

9. Watson, C. T. et al. Genome-wide DNA methylation profiling in the superior temporal gyrus reveals epigenetic signatures associated with Alzheimer's disease. Genome Med. 8, 5 (2016).

10. Lardenoije, R. et al. Alzheimer's disease-associated (hydroxy)methylomic changes in the brain and blood. Clin. Epigenetics 11, 164 (2019).

11. Gasparoni, G. et al. DNA methylation analysis on purified neurons and glia dissects age and Alzheimer's disease-specific changes in the human cortex. Epigenetics Chromatin 11, 41 (2018).
12. Brokaw, D. L. et al. Cell death and survival pathways in Alzheimer's disease an integrative hypothesis testing approach utilizing -omic data sets. Neurobiol. Aging 95, 15-25 (2020).

13. Smith, A. R. et al. A cross-brain regions study of ANK1 DNA methylation in different neurodegenerative diseases. Neurobiol. Aging 74, 70-76 (2019).

14. Nyholt, D. R. A simple correction for multiple testing for single-nucleotide polymorphisms in linkage disequilibrium with each other. Am. J. Hum. Genet 74, 765-769 (2004).

15. Shireby, G. L. et al. Recalibrating the epigenetic clock: implications for assessing biological age in the human cortex. Brain 143, 3763-3775 (2020).

16. Slieker, R. C. et al. Identification and systematic annotation of tissue-specific differentially methylated regions using the Illumina $450 \mathrm{k}$ array. Epigenetics Chromatin 6, 26 (2013).

17. $\mathrm{Ng}, \mathrm{B}$. et al. An xQTL map integrates the genetic architecture of the human brain's transcriptome and epigenome. Nat. Neurosci. 20, 1418-1426 (2017).

18. Kunkle, B. W. et al. Genetic meta-analysis of diagnosed Alzheimer's disease identifies new risk loci and implicates Abeta, tau, immunity and lipid processing. Nat. Genet. 51, 414-430 (2019).

19. Selkoe, D. J. The molecular pathology of Alzheimer's disease. Neuron 6 487-498 (1991).

20. $\mathrm{Xu}$, J. et al. Regional protein expression in human Alzheimer's brain correlates with disease severity. Commun. Biol. 2, 43 (2019).

21. Labadorf, A. et al. RNA sequence analysis of human Huntington disease brain reveals an extensive increase in inflammatory and developmental gene expression. PLoS ONE 10, e0143563 (2015)

22. Roubroeks, J. A. Y. et al. An epigenome-wide association study of Alzheimer's disease blood highlights robust DNA hypermethylation in the HOXB6 gene. Neurobiol. Aging 95, 26-45 (2020).

23. Friedrich, J. et al. Hox function is required for the development and maintenance of the drosophila feeding motor unit. Cell Rep. 14, 850-860 (2016).

24. Sun, L. L., Yang, S. L., Sun, H., Li, W. D. \& Duan, S. R. Molecular differences in Alzheimer's disease between male and female patients determined by integrative network analysis. J. Cell Mol. Med. 23, 47-58 (2019).

25. Rosenberger, A. F. et al. Protein kinase activity decreases with higher braak stages of Alzheimer's disease pathology. J. Alzheimers Dis. 49, 927-943 (2016).

26. Satoh, J., Tabunoki, H., Ishida, T., Saito, Y. \& Arima, K. Accumulation of a repulsive axonal guidance molecule RGMa in amyloid plaques: a possible hallmark of regenerative failure in Alzheimer's disease brains. Neuropathol. Appl. Neurobiol. 39, 109-120 (2013).

27. Fernandez, A. F. et al. A DNA methylation fingerprint of 1628 human samples. Genome Res. 22, 407-419 (2012).

28. Masliah, E., Dumaop, W., Galasko, D. \& Desplats, P. Distinctive patterns of DNA methylation associated with Parkinson disease: identification of concordant epigenetic changes in brain and peripheral blood leukocytes. Epigenetics 8, 1030-1038 (2013).

29. Sanchez-Mut, J. V. et al. Human DNA methylomes of neurodegenerative diseases show common epigenomic patterns. Transl. Psychiatry 6, e718 (2016).

30. MacBean, L. F., Smith, A. R. \& Lunnon, K. Exploring beyond the DNA sequence: a review of epigenomic studies of DNA and histone modifications in dementia. Curr. Genet. Med. Rep. 8, 79-92 (2020).

31. Relton, C. L. \& Smith, Davey G. Mendelian randomization: applications and limitations in epigenetic studies. Epigenomics 7, 1239-1243 (2015).

32. Beach, T. G. et al. Arizona study of aging and neurodegenerative disorders and brain and body donation program. Neuropathology 35, 354-389 (2015).

33. R Development Core Team. R: A Language and Environment for Statistical Computing. R Foundation for Statistical Computing, Vienna, Austria 2012 (2012).

34. Gentleman, R. C. et al. Bioconductor: open software development for computational biology and bioinformatics. Genome Biol. 5, R80 (2004).

35. Aryee, M. J. et al. Minfi: a flexible and comprehensive Bioconductor package for the analysis of Infinium DNA methylation microarrays. Bioinformatics 30, 1363-1369 (2014)

36. Pidsley, R. et al. A data-driven approach to preprocessing Illumina $450 \mathrm{~K}$ methylation array data. BMC Genomics 14, 293 (2013).

37. Chen, Y. A. et al. Discovery of cross-reactive probes and polymorphic CpGs in the Illumina Infinium HumanMethylation450 microarray. Epigenetics 8, 203-209 (2013).

38. Price, M. E. et al. Additional annotation enhances potential for biologicallyrelevant analysis of the Illumina Infinium HumanMethylation450 BeadChip array. Epigenetics Chromatin 6, 4 (2013).

39. Guintivano, J., Aryee, M. J. \& Kaminsky, Z. A. A cell epigenotype specific model for the correction of brain cellular heterogeneity bias and its application to age, brain region and major depression. Epigenetics 8, 290-302 (2013).

40. Gusel'nikova, V. V. \& Korzhevskiy, D. E. NeuN as a neuronal nuclear antigen and neuron differentiation marker. Acta Nat. 7, 42-47 (2015). 
41. Chakraborty, S., Datta, S. \& Datta, S. Surrogate variable analysis using partial least squares (SVA-PLS) in gene expression studies. Bioinformatics 28, 799-806 (2012).

42. van Iterson, M., van Zwet, E. W., Consortium, B. \& Heijmans, B. T. Controlling bias and inflation in epigenome- and transcriptome-wide association studies using the empirical null distribution. Genome Biol. 18, 19 (2017).

43. Schwarzer, G. meta: A R package for meta-analysis. R. News 7, 40-45 (2007).

44. Pinheiro, J., Bates, D., DebRoy, S. \& Sarkar, D. nlme: Linear and Nonlinear Mixed Effects Models (2019).

45. McLean, C. Y. et al. GREAT improves functional interpretation of cisregulatory regions. Nat. Biotechnol. 28, 495-501 (2010).

46. Phipson, B., Maksimovic, J. \& Oshlack, A. missMethyl: an R package for analyzing data from Illumina's HumanMethylation450 platform. Bioinformatics 32, 286-288 (2016).

47. Supek, F., Bosnjak, M., Skunca, N. \& Smuc, T. REVIGO summarizes and visualizes long lists of gene ontology terms. PLoS ONE 6, e21800 (2011).

48. Pedersen, B. S., Schwartz, D. A., Yang, I. V. \& Kechris, K. J. Comb-p: software for combining, analyzing, grouping and correcting spatially correlated Pvalues. Bioinformatics 28, 2986-2988 (2012).

49. Friedman, J. et al. glmnet: Lasso and Elastic-Net Regularized Generalized Linear Models. (2019).

50. Smith, R. G. et al. A meta-analysis of epigenome-wide association studies in Alzheimer's disease highlights novel differentially methylated loci across cortex. GitHub (https://zenodo.org/badge/latestdoi/227141686) (2021).

\section{Acknowledgements}

This work was funded by a major project grant from the Alzheimer's Society UK (ASPG-14-038) to K.L., an Alzheimer's Association US New Investigator Research Grant (NIRG-14-320878) to K.L. and a project grant from the Medical Research Council (MRC) (MR/N027973/1) to K.L. as part of a larger collaborative project funded to K.L. and D.L.A.v.d.H. for the EPI-AD consortium through the Joint Programme-Neurodegenerative Disease Research (JPND) initiative. This work was also supported through a ZonMw Memorabel Grant (733050516) to E.P. Data analysis was undertaken using high-performance computing supported by a Medical Research Council (MRC) Clinical Infrastructure Award (M008924) to J.M. The project was also supported through $\mathrm{PhD}$ studentships from the Alzheimer's Society (G.S.), BRACE (Bristol Research into Alzheimer's and Care of the Elderly) (G.W.) and the MRC GW4 Doctoral Training Program (DTP) (J.A.Y.R.). BDR is jointly funded by Alzheimer's Research UK and the Alzheimer's Society in association with the MRC. DNA methylation data generated in the Brains for Dementia Research cohort was supported by the Alzheimer's Society and Alzheimer's Research UK (ARUK). Research reported in this publication was also supported by the National Institute of Aging of the National Institutes of Health under award numbers P30AG19610, P30AG10161, R01AG15819, R01AG17917, R01AG36042, R01AG036039, R01AG036400 and R01AG067015. The content is solely the responsibility of the authors and does not necessarily represent the official views of the National Institutes of Health We thank the donors and families who have made this research possible.

\section{Author contributions}

A.R.S. and G.W. conducted laboratory experiments. R.G.S., E.P., G.S., E.H., W.V. and M.W. undertook data analysis, bioinformatics and/or support with data review. R.G.S., E.P., A.R.S., J.A.Y.R., D.M., G.G., M.R., A.G., A.J.S., L.S., V.H., D.L.A.v.d.H., D.B., P.T.F., A.J.T., S.L., K.M., J.W., P.D.C., D.A.B., P.L.D.J., J.M. and K.L. provided data for the metaanalysis. L.S. developed the online database. K.L. conceived of the idea and directed the project. K.L., R.G.S. and E.P. drafted the manuscript. All authors read and approved the final submission.

\section{Competing interests}

The authors declare no competing interests.

\section{Additional information}

Supplementary information The online version contains supplementary material available at https://doi.org/10.1038/s41467-021-23243-4.

Correspondence and requests for materials should be addressed to K.L.

Peer review information Nature Communications thanks Dan Arking and the other, anonymous, reviewer(s) for their contribution to the peer review of this work.

Reprints and permission information is available at http://www.nature.com/reprints

Publisher's note Springer Nature remains neutral with regard to jurisdictional claims in published maps and institutional affiliations.

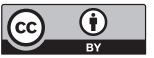

Open Access This article is licensed under a Creative Commons Attribution 4.0 International License, which permits use, sharing, adaptation, distribution and reproduction in any medium or format, as long as you give appropriate credit to the original author(s) and the source, provide a link to the Creative Commons license, and indicate if changes were made. The images or other third party material in this article are included in the article's Creative Commons license, unless indicated otherwise in a credit line to the material. If material is not included in the article's Creative Commons license and your intended use is not permitted by statutory regulation or exceeds the permitted use, you will need to obtain permission directly from the copyright holder. To view a copy of this license, visit http://creativecommons.org/ licenses/by/4.0/.

(C) The Author(s) 2021 